\title{
Evaluation of routine Isoniazid Preventive Therapy among People Living with HIV for reducing Tuberculosis; a retrospective cohort study in Tanzania (2012-2016)
}

\section{Werner Maokola ( $\nabla$ drwernerm@yahoo.com )}

Kilimanjaro Christian Medical University College https://orcid.org/0000-0003-0782-0784

\section{Bernard Ngowi}

NIMR-Mbeya Medical Research Programme

\section{Lovett Lawson}

Zankli Medical Centre

Michael Mahande

Kilimanjaro Christian Medical University College

Jim Todd

London School of Hygiene and Tropical Medicine Department of Population Health

\section{Sia Msuya}

Kilimanjaro Christian Medical University College

\section{Research article}

Keywords: Tanzania, Isoniazid Preventive Therapy, PLHIV and Tuberculosis

Posted Date: March 31st, 2020

DOI: https://doi.org/10.21203/rs.3.rs-19503/v1

License: (c) (i) This work is licensed under a Creative Commons Attribution 4.0 International License.

Read Full License 
Evaluation of routine Isoniazid Preventive Therapy among People Living with HIV for reducing Tuberculosis; a retrospective cohort study in Tanzania (2012-2016)

Werner Maokola ${ }^{1,2 * * *}$, Bernard Ngowi $^{3}$, Lovetti Lawson ${ }^{4}$, Michael Mahande ${ }^{2}$, Jim Todd $^{5,6}$ and Sia Msuya ${ }^{2}$

${ }^{1}$ Ministry of Health, Community Development, Gender, Elderly and Children-Tanzania

${ }^{2}$ Institute of Public Health, Kilimanjaro Christian Medical University College Moshi Tanzania, ${ }^{3}$ Mbeya University College of Health Sciences-Tanzania' ${ }^{4}$ Zankli Medical Center, Abuja Nigeria, ${ }^{5}$ National Institute of Medical Research Mwanza Tanzania' ${ }^{6}$ London School of Hygiene and Tropical Medicine United Kingdom'

****Correspondence: $\underline{\text { drwernerm @ yahoo.com }}$ 


\section{ABSTRACT:}

Background: Isoniazid Preventive Therapy (IPT) reduced Tuberculosis (TB) among People Living with HIV (PLHIV). Despite this, uptake has been reported to be suboptimal. We describe characteristics of visits in which PLHIV were screened TB negative (as the main source for IPT initiation), determine characteristics of visits in which PLHIV were initiated on IPT as well as determined factors associated with IPT initiation to inform program scale up and improve quality of service.

Methods: Retrospective cohort study design which involved PLHIV enrolled into care and treatment clinics in Dar es Salaam, Iringa and Njombe regions from January 2012 to December 2016. The study aimed at evaluating implementation of IPT among PLHIV. Data analysis was conducted using STATA.

\section{Results:}

A total 173,746 were enrolled in CTC in the 3 regions during the period of follow up and made a total of $2,638,876$ visits. Of the eligible visits, only $24,429(1.26 \%)$ were initiated on IPT.

In multivariate analysis, 50 years and more (aOR=3.42, 95\% CI: 3.07-3.82, $\mathrm{P}<0.01$ ), bedridden functional status individuals with bedridden functional status ( $\mathrm{aOR}=4.56,95 \%$ CI:2.45-8.49, P<0.01) and WHO clinical stage II had higher odds of IPT initiation $(\mathrm{aOR}=1.18,95 \% \mathrm{CI}: 1.13-1.23, \mathrm{P}<0.01)$. Furthermore, enrolment in $2016(\mathrm{aOR}=2.92$, 95\% CI:2.79-3.06, $\mathrm{P}<0.01)$, enrolment in hospitals $(\mathrm{aOR}=1.84$, 95\% CI:1.77-1.90, $\mathrm{P}<0.01)$, enrolment in public health facilities $(\mathrm{aOR}=1.82,95 \% \mathrm{CI}: 1.75-1.90, \mathrm{P}<0.01)$ and been on care for more than one year $(\mathrm{aOR}=6.77,95 \% \mathrm{CI}: 5.25-8.73, \mathrm{P}<0.000)$ were also more likely to be initiated on IPT. Enrolment in Iringa (aOR=0.44, 95\% CI: 0.41-0.47, $\mathrm{P}<0.01)$ and good adherence $(\mathrm{aOR}=0.56,95 \% \mathrm{CI} 0.47-0.67, \mathrm{P}<0.000)$ were less likely to be initiated on IPT.

\section{Conclusions:}


Our study documented low IPT initiation proportion among those who were enrolled in HIV care and eligible in the 3 regions during the study period. Variations in IPT initiation among regions signals different dynamics affecting IPT uptake in different regions and hence customized approaches in quality improvement. Implementation research is needed to understand health system as well as cultural barriers in the uptake of IPT intervention.

Key words: Tanzania, Isoniazid Preventive Therapy, PLHIV and Tuberculosis

\section{BACKGROUND:}


Sustainable Development Goals (SDG) and Joint United Nations Programme on HIV/AIDS (UNAIDS) have laid goals and targets to end AIDS epidemic by 2030(1). One of the targets to achieve 2030 HIV targets is to achieve HIV viral suppression among People Living on HIV (PLHIV) who are on Antiretroviral Therapy (ART)(1).Tuberculosis (TB) among PLHIV is still a public health concern(2) which despite wide coverage of ART and other interventions to reduce TB among PLHIV, cause increased ill-health and death(3), thus posing threats towards achieving SDG goals. Worldwide, in 2018, $9 \%$ of individuals diagnosed with TB disease were HIV positive, $51 \%$ of those with HIV had Tuberculosis disease with84\% on ART. Death among diagnosed TB was 1.3 million, among these 300,000 (23\%) were HIV positive(2).

Isoniazid Preventive Therapy (IPT) which entails use of an anti-TB Drug called Isoniazid (INH) for at least 6 months, treats latent TB infection thus preventing the development of active TB disease in high risk population like PLHIV(4). IPT in PLHIV is one of the proven public health interventions to reduce TB disease among PLHIV(5)(6)(7).Following this, World Health Organization (WHO) recommends IPT be part of comprehensive HIV care and treatment package(8).

Despite available evidence on the benefits of IPT on PLHIV, use of this effective preventive tool is suboptimal(9)(10)(11)(12). Suboptimal implementation of IPT is equally present even in parts of the World hard-hit by both HIV and TB like in SubSaharan Africa(13) where service is needed most. In 2017, IPT among PLHIV Worldwide ranged from $1 \%$ in Eswatini to $53 \%$ in South Africa(2). Reasons for low IPT coverage ranges from health provider factors, client factors to general health system factors(9)(14).

Tanzania has been implementing IPT among PLHIV in HIV care and treatment clinics to prevent development of TB diseases since 2011 (15). Here we describe characteristics of PLHIV who were screened TB negative (prerequisite for IPT initiation), determine characteristics of PLHIV who initiated IPT as well as determine factors associated with 
IPT initiation. The findings from this paper will inform IPT program scale up in Tanzania and elsewhere.

\section{METHODS:}

\section{Study design and setting:}

We conducted a retrospective cohort study design which involved PLHIV enrolled in 315 HIV care and treatment clinics (CTCs) in Dar es Salaam, Iringa and Njombe regions from January 2012 to December 2016. The study involved secondary data analysis of deidentified PLHIV from these three regions in Tanzania. Iringa and Njombe regions have been documented to have higher HIV prevalence above national average(16) and Dar es Salaam to have higher TB case notifications in the country (17).

\section{Study Population and Data collection:}

The study used retrospective data collected from PLHIV enrolled in HIV care and treatment clinics in the three regions enrolled in the specified study period. In Tanzania TB management is integrated in HIV care and treatment services to reduce TB among PLHIV. Information regarding the cohort is detailed elsewhere (18). In summary, three interventions to reduce TB are implemented in HIV care and treatment clinics; these are Intensified TB case finding (ICF), IPT and TB infection prevention and control. During every clinic visit PLHIV are screened for active TB using symptoms/signs as part of ICF. Those who are found with at least one symptom or sign of TB undergo further laboratory and radiological investigations to diagnose TB disease. Those who are found to have no symptoms/signs of active TB are offered IPT for at least 6 months provided other criteria are fulfilled. TB infection and prevention measures are also implemented in all HIV care and treatment clinics to reduce TB infection transmission.

CTCs have patient-level data collection containing data on every visit made by PLHIV. For up to $80 \%$ of clinics, data including those related to TB are electronically captured at health facility level into an electronic database called the CTC2 database. Data from the CTC2 database is transferred to national level database (CTC3) in real time, where 
demographic and clinical information of each PLHIV identified only by their unique identification CTC number is available for analysis.

Information for PLHIV enrolled in HIV care and treatment clinics in Dar es Salaam, Iringa and Njombe regions were extracted from the CTC3 database. The extracted database had the following variables: patient unique ID, CTC enrolment year, Age, Date of birth, sex, health facility level, clinic visit dates, CD4, WHO staging, Visit number, TB screening, TB treatment/IPT and ART status.

The data were cleaned by checking missing values, abnormal entries. STATA version 14 was used for data analysis. Demographic and clinical variables were used as independent variables. Visits with corresponding TB screening status (positive/negative TB screening) and IPT status (received/not received) were the dependent variables.

Descriptive analysis was used to describe baseline characteristics of PLHIV with negative TB screening. Multivariate logistic regression was used to determine factors associated with negative TB screening and IPT initiation status involving only exposure variables which were statistical significant during univariate analysis. Multilevel analysis was done to account for cluster effects at health facility. Statistical significance level of 5\% was taken as statistical significance level.

\section{RESULTS:}

Characteristics of study participants: A total 173,746 PLHIV were enrolled in CTC in the 3 regions during the period from 2012 to 2016. Over the 5 years the cohort made a total of 2,638,876 visits, for an average of 15.2 visits per PLHIV. Of these visits, 1,944,230 (73.68\%) were eligible for IPT eligibility assessment. A total of 694,646 visits (26.32\%) were excluded from the analysis as they were either from health facilities not implementing IPT at some point in the study period or had visits involving TB treatment related or were visits corresponding to less than 2 years from IPT initiation. Among IPT eligible visits, IPT was initiated in 24,429 (1.26\%) (Figure 1). 
When compared to positive TB screening visits, negative TB negative visits were more likely to be those of females (69.93\%), age group 25-49 years $(73.41 \%)$, bedridden functional status $(99.03 \%)$ and those on ARV (86.93\%). Negative TB screening was also more in visits with WHO stage III (43.44\%), those enrolled in 2012 (26.12\%) and those enrolled in hospitals (36.38\%). Negative TB screening visits was also more in public health facilities (72.67\%), enrolled in Dar es Salaam region (67.55\%), those with good ARV adherence (98.82\%) and visits beyond one year after CTC enrolment $(78.06 \%)$ (Table 1).

Baseline characteristics of IPT initiation visits: Visits involving females $(69.91 \%)(\mathrm{P}<0.001)$, those aged 25-49 years $(73.36 \%)(\mathrm{P}<0.001)$, bedridden functional status $(99.02 \%)(\mathrm{P}<0.001)$ and on ART $(86.80 \%)(\mathrm{P}<0.001)$ were more likely to be initiated on IPT. IPT initiation was also more common for visits with WHO stage III (43.41\%) ( $\mathrm{P}<0.001)$, enrolment in 2014 (26.21\%) ( $<<0.001)$, attending hospitals $(36.43 \%)$ $(\mathrm{P}<0.001)$, attending public health facilities $(72.52 \%)(\mathrm{P}<0.001)$, enrolled in Dar es Salaam region $(67.45 \%)(\mathrm{P}<0.001)$, having good adherence $(98.81 \%)(\mathrm{P}<0.001)$ and in care for more than one year $(91.19 \%)(\mathrm{P}<0.001)$ (Table 2$)$.

Determinants of IPT initiation: In multivariate analysis; age, functional status, WHO Clinical stage, enrolment year, health facility type, health facility ownership, region, ARV adherence status and visit number had statistical significant association with IPT initiation. Fifty (50) years and more was 3 times more likely to be initiated on IPT than younger ages $(\mathrm{aOR}=3.42,95 \% \mathrm{CI}: 3.07-3.82, \mathrm{P}<0.01)$, bedridden functional status was up to 4 times more likely to be initiated on IPT compared to walking ( $\mathrm{aOR}=4.56,95 \% \mathrm{CI}: 2.45-8.49$, $\mathrm{P}<0.01)$ and WHO clinical stage II was $72 \%$ more likely to be initiated on IPT than other WHO clinical stages $(\mathrm{aOR}=1.18,95 \% \mathrm{CI}: 1.13-1.23, \mathrm{P}<0.01)$. Furthermore, enrolment in 2016 was about 3 times more likely to be initiated on IPT than in other years before 2016 $(\mathrm{aOR}=2.92$, 95\% CI:2.79-3.06, $\mathrm{P}<0.01)$, enrolment in hospitals was 2 times more likely to be initiated than those in lower health facilities $(\mathrm{aOR}=1.84,95 \% \mathrm{CI}: 1.77-1.90, \mathrm{P}<0.01)$, enrolment in public health facilities was about 2 times more likely to be initiated than 
those in private $(\mathrm{aOR}=1.82,95 \% \mathrm{CI}: 1.75-1.90, \mathrm{P}<0.01)$ and been on care for more than one year was close to 7 times more likely to be initiated on IPT than those on care less than a year $(\mathrm{aOR}=6.77,95 \% \mathrm{CI}: 5.25-8.73, \mathrm{P}<0.01)$. Enrolment in Iringa region was up to $56 \%$ less likely to be initiated on IPT than in Dar es Salaam (aOR=0.44, 95\% CI: 0.41$0.47, \mathrm{P}<0.01)$ and good ARV adherence was less likely to be initiated by $44 \%$ compared with poor ARV adherence $(\mathrm{aOR}=0.56,95 \%$ CI 0.47-0.67, $\mathrm{P}<0.01)$ (Table 3$)$.

\section{DISCUSSION:}

This retrospective cohort study registered 173,746 PLHIV who made a total of 2, 638,876 visits from January 2012 to December 2016 enrolled in 315 CTCs in Dar es Salaam, Iringa and Njombe regions. The study documented high proportion of IPT eligible visits with low initiation of IPT.

Our study observed a high IPT eligibility proportions among visits with negative TB screening. High IPT eligibility has also been documented in others studies (19)(20). However, our study registered lower IPT initiation, unlike other studies which were also conducted in routine settings in Zimbabwe, Ethiopia and Nepal (12)(21)(5)(22)(23)(24). These studies documented IPT initiation ranging from $20 \%$ to $54 \%$. The observed low IPT initiation in the current study needs further research. Nevertheless, most of the studies, despite being conducted in routine settings like our study, involved small numbers of health facilities. None of the above studies in Zimbabwe, Ethiopia and Nepal $(12)(21)(5)(22)(23)(24)$ involved more than 20 high level health facilities. The study under discussion involved a total of 315 in the 3 regions containing a mix of high and low level health facilities. Furthermore, our study analyzed the likelihood for IPT initiation among individual eligible visits in the cohort, unlike the other studies which considered individuals. Shortage of INH, fear of INH resistance, lack of confidence in ruling out TB disease, lack of commitment among providers and client acceptance were among the barriers for IPT implementation documented in several studies (21)(22)(25).

Consistent with our study, other studies also documented a high IPT initiation proportion among females, adults aged between 24 and 49, ART recipients and PLHIV with good 
ARV adherence (21)(26) (27)(28). High uptake among females could be due to more health information to women during medical encounter such as maternal and child health services (25). ART experience and good ARV adherence may increase confidence for IPT initiation among health care providers.

In our study IPT initiation was associated with advanced age above 50, being bedridden, WHO clinical stage II and enrolment into care in 2016. Other determinants were enrolment in hospitals, enrolment in public health facilities and having been in care for more than one year. IPT initiation among elder population was also reported in another study (24). Tendency to provide IPT to elderly population could be due to perceived risk of TB diseases by health care providers for elderly population. We found IPT initiation to be associated in visits involving health individuals (WHO clinical stage II). Our finding is agreement with what was found in Kenya (21). Reason for avoiding IPT in severely ill PLHIV can be attributed to fear of clinicians due to difficulty in ruling out TB disease in this population as speculated in the Kenyan study (21). High IPT initiation in hospitals and public health facilities could be explained by the fact that IPT was rolled out gradual starting with Hospitals. Higher initiation in public versus in private health facilities could be due to small pace of private health facilities in implementing new policies (29). Visits corresponding to being in care for more than one year had higher odds of IPT initiation than earlier visits. IPT initiation later after HIV care enrolment is also reported in Nepal (24). Lower IPT initiation associated with Iringa compared to Dar es Salaam and Njombe explains dynamics in IPT implementation which also differs from health facility to health facility as reported in another study (24). Lower IPT initiation in visits with good adherence is contrary to what is expected in normal program settings as good ARV adherence was one of the criteria for IPT initiation. We expected IPT to be initiated more readily in PLHIV with good ARV adherence than in those with poor adherence. This finding needs to be supplemented by qualitative evaluation.

Our study had several limitations. Firstly, the data used for the analysis is routinely collected from HIV program in Tanzania. As collection happens in diverse of health 
facilities including high and low level, the quality of data collection may also vary and hence affect the data analysis. Secondly, only about $80 \%$ of the HIV clinics in the country had electronic HIV database and hence capable of contributing data to the national database. Health facilities at low level did not have electronic database and were unable to contribute data. Thirdly, the analysis included 3 regions out of 26 regions. These regions were purposively chosen as they had highest HIV and TB prevalence. They may hence not represent a true of picture present in other regions which were not involved in the analysis.

\section{CONCLUSION:}

Our study which used routine HIV data from 315 clinics documented low IPT initiation proportion among those who were enrolled in HIV care and eligible for IPT in Dar es Salaam, Iringa and Njombe during the study period. Variations in IPT initiation among regions signal different dynamics affecting IPT uptake in different regions and hence require customized approaches in quality improvement. Implementation research is needed to understand health system as well as cultural barriers in the uptake of IPT intervention and design quality improvement initiative accordingly.

\section{List of abbreviations:}

ART-Antiretroviral Therapy

ARV-Antiretroviral drugs

$\mathrm{CTC}=$ Care and Treatment Clinic

DTA=Data Transfer Agreement

ICF=Intensified TB Case Finding

$\mathrm{INH}=$ Isoniazid

IPT=Isoniazid Preventive Therapy 
MoHCDGEC=Ministry of Health, Community Development, Gender, Elderly and Children

PLHIV=People Living with HIV

SDG=Sustainable Development Goals

$\mathrm{TB}=$ Tuberculosis

UNAIDS=United Nations Joint Programme on HIV/AIDS

WHO=World Health Organization

\section{Declarations:}

This article is an original research which is one of the required number of published articles for $\mathrm{PhD}$ training of the first author. We declare that this article has not been submitted to any other journal for publication.

\section{Ethics approval and consent to participate:}

The study used routine HIV data from Tanzania. Hence, the study did not have contact with human subjects. Approval for conducting the study was obtained from Kilimanjaro Christian Medical University College in Moshi Tanzania. Permission to use routine HIV data was obtained from Ministry of Health, Community Development, Gender, Elderly and Children (MoHCDGEC) Tanzania by signing Data Transfer Agreement (DTA).

\section{Consent for publication:}

Authors of the study collaboratively give permission to the publisher to publish the study findings as presented by the authors.

\section{Availability of data and materials:}


Data and other materials used for the study belong to the MoHCDGEC Tanzania. Permission to access data and other materials used for the study can be obtained from the Permanent Secretary MoHCDGE in Tanzania.

\section{Competing interests:}

Authors of the study declare that there is no conflict of interest involved in conducting the study.

\section{Funding:}

The study was supported by the SEARCH (Sustainable Evaluation through Analysis of Routinely Collected HIV data) Project. We acknowledge funding by the Bill \& Melinda Gates Foundation grant number OPP1084472 entitled "Using routinely collected public facility data for program improvement in Tanzania, Malawi and Zambia." This paper has been produced as part of the PhD studies of WM for the evaluation of TB services in HIV care and treatment services in Tanzania was financed collaboratively by the Government of Tanzania and US President's Emergency Plan for AIDS Relief (PEPFAR).

\section{Authors' contributions:}

WM conceived the study idea, developed first draft of the study protocol, developed data analysis plan and developed the first draft of the manuscript. BN, LL, JT, MM and SM reviewed study protocol, data analysis plan and study manuscript. WM, BN, LL, JT, MM and SM conducted data management. All authors agreed on the final version of the manuscript.

\section{Acknowledgements:}

We would like to send our gratitude to Bill and Melinda Gates Foundation, PEPFAR and the Government of Tanzania for financial support. We also thank staff within National AIDS Control Program/MoHCDGEC Tanzania as well as staff from Regional, Council and health facility levels for their contribution in making sure that routine HIV data collected is of quality. 


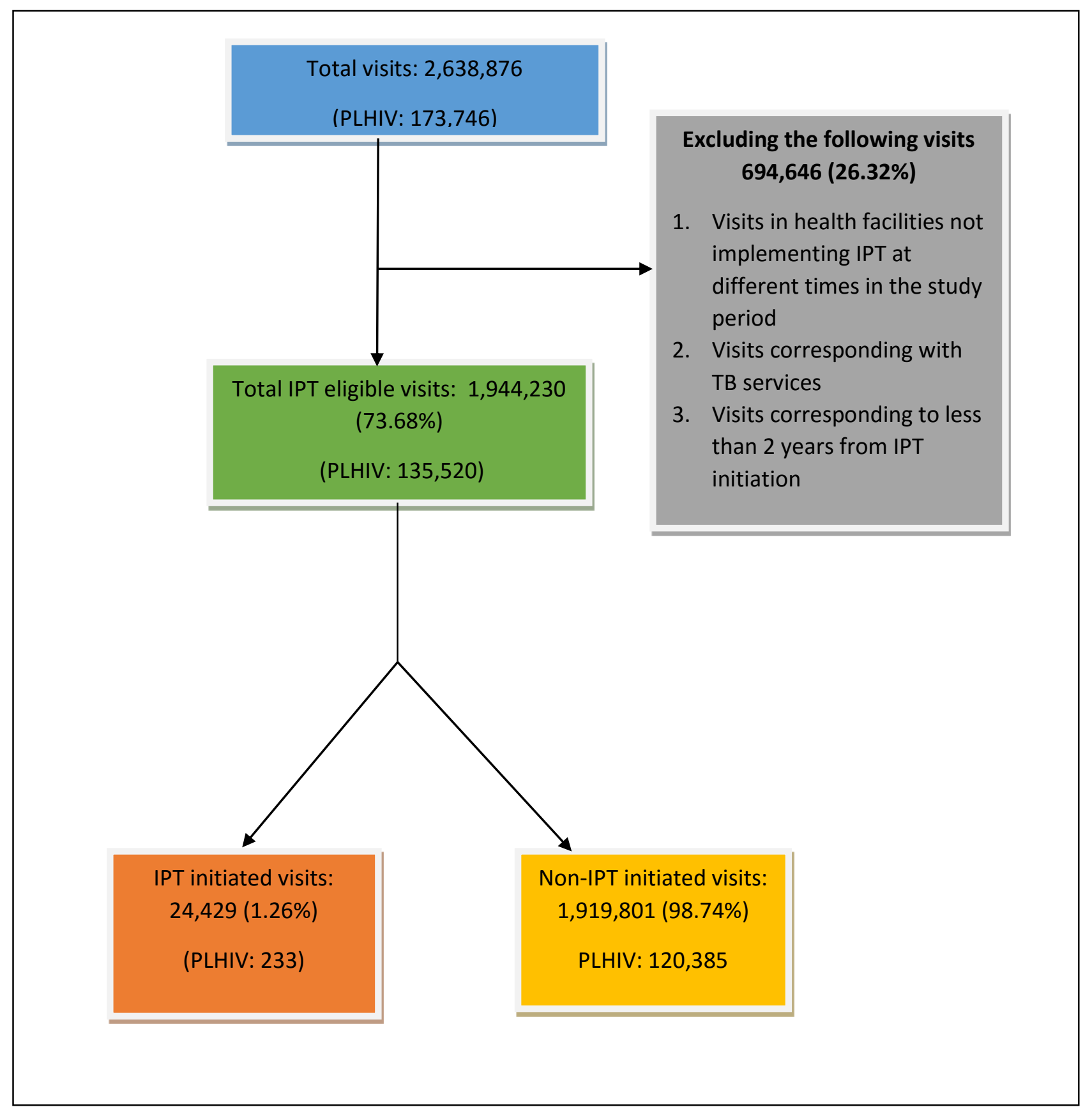

Figure 1: IPT initiation cascade among PLHIV attending CTC during the follow up period 
Table 1: Baseline characteristics of study participants' visits:

\begin{tabular}{|c|c|c|c|}
\hline \multirow[t]{2}{*}{ Variable } & \multicolumn{3}{|c|}{ TB screening status (visits) } \\
\hline & $\begin{array}{l}\text { Positive (n, \%) } \\
n=46,044\end{array}$ & $\begin{array}{l}\text { Negative (n, \%) } \\
\text { n=1,944,230 }\end{array}$ & $\begin{array}{l}\text { Total } \\
\text { N=1,990,534 }\end{array}$ \\
\hline Sex: Male & $16,84(36.57 \%)$ & $584,685(30.07 \%)$ & $601,525(30.22 \%)$ \\
\hline Female & $29,204(63.43 \%)$ & $1,359,545(69.93 \%)$ & $1,388,749(69.78 \%)$ \\
\hline \multicolumn{4}{|l|}{ Age: } \\
\hline $0-9$ & $3,300(7.17 \%)$ & $94,935(4.88 \%)$ & $98,253(4.94 \%)$ \\
\hline $10-19$ & $2,369(5.15 \%)$ & $80,914(4.16 \%)$ & $83,283(4.18 \%)$ \\
\hline $20-24$ & $1,973(4.29 \%)$ & $118,081(6.07 \%)$ & $120,054(6.03 \%)$ \\
\hline $25-49$ & $32,301(70.18 \%)$ & $1,427,190(73.41 \%)$ & $1,459,491(73.33 \%)$ \\
\hline $50+$ & $6,083(13.22 \%)$ & $223,060(11.47 \%)$ & $229,143(11.51 \%)$ \\
\hline \multicolumn{4}{|c|}{ Functional status: } \\
\hline Ambulatory & $2,442(5.31 \%)$ & $12,733(0.66 \%)$ & $15,175(0.76 \%)$ \\
\hline Bedridden & $609(1.32 \%)$ & $6,175(0.32 \%)$ & $6,784(0.34 \%)$ \\
\hline Walking & $42,927(93.36 \%)$ & $1,924,138(99.03 \%)$ & $1,967,065(98.90 \%)$ \\
\hline \multicolumn{4}{|l|}{ ART groups } \\
\hline Not on ART & $15,934(34.95 \%)$ & $254,091(13.07 \%)$ & $270,025(13.57 \%)$ \\
\hline On ART & $29,658(65.05 \%)$ & $1,689,631(86.93 \%)$ & $1,719,289(86.43 \%)$ \\
\hline \multicolumn{4}{|c|}{ WHO clinical staging } \\
\hline I & $4,060(9.40 \%)$ & $478,562(24.79 \%)$ & $482,622(24.45 \%)$ \\
\hline II & $8,943(20.71 \%)$ & $435,165(22.54 \%)$ & $444,108(22.50 \%)$ \\
\hline III & $24,563(56.87 \%)$ & $838,821(43.44 \%)$ & $863,384(43.74 \%)$ \\
\hline IV & $5,624(13.02 \%)$ & $178,277(9.23 \%)$ & $183,901(9.32 \%)$ \\
\hline \multicolumn{4}{|c|}{ Enrolment year } \\
\hline 2012 & $14,333(31.13 \%)$ & $507,747(26.12 \%)$ & $522,080(26.23 \%)$ \\
\hline 2013 & $13,040(28.32 \%)$ & $501,119(25.77 \%)$ & $514,159(25.83 \%)$ \\
\hline
\end{tabular}




\begin{tabular}{|c|c|c|c|}
\hline 2014 & $8,185(17.78 \%)$ & $430,642(22.15 \%)$ & $438,827(22.05 \%)$ \\
\hline 2015 & $5,585(12.13 \%)$ & $287,641(14.79 \%)$ & $293,226(14.73 \%)$ \\
\hline 2016 & $4,901(10.64 \%)$ & $217,081(11.17 \%)$ & $221,982(11.15 \%)$ \\
\hline \multicolumn{4}{|c|}{ Health Facility level } \\
\hline Dispensary & $13,994(30.39 \%)$ & $642,085(33.03 \%)$ & $656,079(32.96 \%)$ \\
\hline Health Center & $7,821(16.99 \%)$ & $590,915(30.39 \%)$ & $598,736(30.08 \%)$ \\
\hline Hospital & $24,229(52.62 \%)$ & $711,230(36.38 \%)$ & $735,459(36.95 \%)$ \\
\hline \multicolumn{4}{|c|}{ Health Facility ownership } \\
\hline Private & $10,613(23.05 \%)$ & $531,367(27.33 \%)$ & $541,980(27.23 \%)$ \\
\hline Public & $35,431(76.95 \%)$ & $1,412,863(72.67 \%)$ & $1,448,294(72.77 \%)$ \\
\hline \multicolumn{4}{|l|}{ Region } \\
\hline Dar es Salaam & $36,765(79.85 \%)$ & $1,313,355(67.55 \%)$ & $1,350,120(67.84 \%)$ \\
\hline Iringa & $6,412(13.93 \%)$ & $188,759(9.71 \%)$ & $195,171(9.81 \%)$ \\
\hline Njombe & $2,867(6.23 \%)$ & $442,116(22.47 \%)$ & $444,983(22.36 \%)$ \\
\hline \multicolumn{4}{|l|}{ Adherence } \\
\hline Good & $23,954(97.88 \%)$ & $1,518,295(98.82 \%)$ & $1,542,249(98.81 \%)$ \\
\hline Poor & $519(2.12 \%)$ & $18,106(1.18 \%)$ & $18,625(1.19 \%)$ \\
\hline \multicolumn{4}{|l|}{ Visit number } \\
\hline First visit & $14,240(30.93 \%)$ & $117,452(6.04 \%)$ & $131,692(6.62 \%)$ \\
\hline Within first year & $11,510(25.00 \%)$ & $308,744(15.88 \%)$ & $320,254(16.09 \%)$ \\
\hline Beyond $1^{\text {st }}$ year & $20,294(44.08 \%)$ & $1,518,034(78.08 \%)$ & $1,538,328(77.29 \%)$ \\
\hline
\end{tabular}

Table 2: Baseline characteristics of IPT initiation visits

\begin{tabular}{|l|l|l|l|l|}
\hline Variable & \multicolumn{2}{|l|}{ IPT initiation status } & Total & P-value \\
\hline & IPT initiated & IPT not initiated & Total & \\
\hline
\end{tabular}




\begin{tabular}{|c|c|c|c|c|}
\hline & $n=24,429$ & $\mathrm{n}=\mathbf{1 , 9 1 9 , 8 0 1}$ & $N=1,944,230$ & \\
\hline \multicolumn{5}{|l|}{ Sex: } \\
\hline Male & $6,986(28.60 \%)$ & $577,699(30.09 \%)$ & $584,685(30.07 \%)$ & \multirow[b]{2}{*}{$\mathrm{P}<0.01$} \\
\hline Female & $17,443(71.40 \%)$ & $1,342,102(69.91 \%)$ & $1,359,545(69.93 \%)$ & \\
\hline \multicolumn{4}{|l|}{ Age } & \\
\hline $0-9$ & $387(1.58 \%)$ & $94,566(4.93 \%)$ & $94,953(4.88 \%)$ & \multirow{5}{*}{$\mathrm{P}<0.01$} \\
\hline $10-19$ & $643(2.63 \%)$ & $80,272(4.18 \%)$ & $80,914(4.16 \%)$ & \\
\hline $20-24$ & $1,167(4.78 \%)$ & $116,914(6.09 \%)$ & $118,081(6.07 \%)$ & \\
\hline $25-49$ & $18,939(77.53 \%)$ & $1,408,251(73.36 \%)$ & $1,427,190(73.41 \%)$ & \\
\hline $50+$ & $3,294(13.48 \%)$ & $219,766(11.45 \%)$ & $223,060(11.47 \%)$ & \\
\hline \multicolumn{4}{|c|}{ Functional status: } & \\
\hline Walking & $17(0.07 \%)$ & $12,716(0.66 \%)$ & $12,733(0.66 \%)$ & \multirow{3}{*}{$\mathrm{P}<0.01$} \\
\hline Ambulatory & $66(0.27 \%)$ & $6,109(0.32 \%)$ & $6,175(0.32 \%)$ & \\
\hline Bedridden & $24,325(99.66 \%)$ & $1,899,813(99.02 \%)$ & $1,924,138(99.03 \%)$ & \\
\hline \multicolumn{4}{|l|}{ ART groups } & \\
\hline No ART & $827(3.39 \%)$ & $253,264(13.20 \%)$ & $254,091(13.07 \%)$ & \multirow[b]{2}{*}{$\mathrm{P}<0.01$} \\
\hline ART & $23,597(96.61 \%)$ & $1,666,034(86.80 \%)$ & $1,689,631(86.93 \%)$ & \\
\hline \multicolumn{4}{|c|}{ WHO clinical stage } & \\
\hline I & $5,551(22.88 \%)$ & $473,011(24.81 \%)$ & $478,562(24.79 \%)$ & \multirow{4}{*}{$\mathrm{P}<0.01$} \\
\hline II & $5,425(22.36 \%)$ & $429,740(22.54 \%)$ & $435,165(22.54 \%)$ & \\
\hline III & $11,206(46.19 \%)$ & $827,615(43.41 \%)$ & $838,821(43.44 \%)$ & \\
\hline IV & $2,077(8.56 \%)$ & $176,200(9.24 \%)$ & $178,277(9.23 \%)$ & \\
\hline \multicolumn{4}{|c|}{ Enrolment year } & \\
\hline 2012 & $4,530(18.54 \%)$ & $503,217(26.21 \%)$ & $507,747(26.12 \%)$ & \multirow{5}{*}{$\mathrm{P}<0.01$} \\
\hline 2013 & $5,056(20.70 \%)$ & $496,063(25.54 \%)$ & $501,119(25.77 \%)$ & \\
\hline 2014 & $5,559(22.76 \%)$ & $425,083(22.14 \%)$ & $430,642(22.15 \%)$ & \\
\hline 2015 & $4,745(19.46 \%)$ & $282,887(14.74 \%)$ & $287,6413(14.79 \%)$ & \\
\hline 2016 & $4,530(18.54 \%)$ & $212,551(11.07 \%)$ & $217,081(11.17 \%)$ & \\
\hline
\end{tabular}




\begin{tabular}{|c|c|c|c|c|}
\hline \multicolumn{4}{|c|}{ Health Facility level } & \multirow{4}{*}{$\mathrm{P}<0.0$} \\
\hline \multirow{2}{*}{$\begin{array}{l}\text { Dispensary } \\
\text { Health Center }\end{array}$} & \multirow{2}{*}{$\begin{array}{l}6,169(25.25 \%) \\
6,448(26.39 \%)\end{array}$} & \multirow{2}{*}{$\begin{array}{l}635,916(33.12 \%) \\
584,467(30.44 \%)\end{array}$} & \multirow{2}{*}{$\begin{array}{l}642,085(33.03 \%) \\
590,915(30.39 \%)\end{array}$} & \\
\hline & & & & \\
\hline Hospital & $11,812(48.35 \%)$ & $699,418(36.43 \%)$ & $711,230(36.58 \%)$ & \\
\hline \multicolumn{4}{|c|}{ Health Facility ownership } & \\
\hline Private & $3,769(15.43 \%)$ & $527,598(27.48 \%)$ & $531,367(27.33 \%)$ & \multirow[b]{2}{*}{$\mathrm{P}<0.0$} \\
\hline Public & $20,660(84.57 \%)$ & $1,392,203(72.52 \%)$ & $1,412,863(72.67 \%)$ & \\
\hline \multicolumn{4}{|l|}{ Region } & \\
\hline Dar es Salaam & $18,462(75.57 \%)$ & $1,294,893(67.45 \%)$ & $1,313,355(67.55 \%)$ & \multirow{3}{*}{$\mathrm{P}<0.01$} \\
\hline Iringa & $966(3.95 \%)$ & $187,793(9.78 \%)$ & $188,759(9.71 \%)$ & \\
\hline Njombe & $5,000(20.47 \%)$ & $437,115(22.77 \%)$ & $442,116(22.40 \%)$ & \\
\hline \multicolumn{4}{|l|}{ Adherence } & \\
\hline Good & $22,242(99.40 \%)$ & $1,496,053(98.81 \%)$ & $1,518,295(98.82 \%)$ & \multirow[b]{2}{*}{$\mathrm{P}<0.01$} \\
\hline Poor & $135(0.60 \%)$ & $17,971(0.60 \%)$ & $18,106(1.18 \%)$ & \\
\hline \multicolumn{4}{|l|}{ Visit number } & \\
\hline First visit & $194(0.79 \%)$ & $117,258(6.11 \%)$ & $117,452(6.04 \%)$ & \multirow{3}{*}{$\mathrm{P}<0.01$} \\
\hline Within first year & $1,958(8.02 \%)$ & $306,786(15.98 \%)$ & $308,744(15.88 \%)$ & \\
\hline Beyond first year & $22,277(91.19 \%)$ & $1,495,757(77.91 \%)$ & $1,518,034(78.08 \%)$ & \\
\hline
\end{tabular}

Table 3: Determinants of visits with IPT initiation: 


\begin{tabular}{|c|c|c|c|c|}
\hline \multirow{2}{*}{\begin{tabular}{|l|} 
\\
Variable
\end{tabular}} & \multicolumn{2}{|l|}{ Crude } & \multicolumn{2}{|l|}{ Adjusted } \\
\hline & $\operatorname{cOR}(95 \%, \mathrm{CI})^{*}$ & P-value & aOR, 95\% CI* & P-value \\
\hline \multicolumn{5}{|l|}{ Sex } \\
\hline Male & 1 & & 1 & \multirow[b]{2}{*}{$\mathrm{P}=0.0333$} \\
\hline Female & $1.07(1.05-1.11)$ & $\mathrm{P}<0.01$ & $1.03(1.00-1.06)$ & \\
\hline \multicolumn{5}{|l|}{ Age groups } \\
\hline $0-9$ & 1 & & 1 & \multirow{5}{*}{$\mathrm{P}<0.01$} \\
\hline $10-19$ & $1.95(1.72-2.22)$ & $\mathrm{P}<0.01$ & $1.88(1.65-2.15)$ & \\
\hline $20-24$ & $2.44(2.17-2.74)$ & $\mathrm{P}<0.01$ & $2.34(2.07-2.64)$ & \\
\hline $25-49$ & $3.29(2.97-3.64)$ & $\mathrm{P}<0.01$ & $3.10(2.80-3.44)$ & \\
\hline+50 & $3.66(3.30-4.07)$ & $\mathrm{P}<0.01$ & 3.42(3.07-3.82) & \\
\hline \multicolumn{5}{|c|}{ Functional status } \\
\hline Walking & 1 & & 1 & \multirow{3}{*}{$\mathrm{P}<0.01$} \\
\hline Ambulatory & $8.08(4.74-13.78)$ & $\mathrm{P}<0.01$ & $3.60(1.85-7.05)$ & \\
\hline Bedridden & $9.58(5.95-15.41)$ & $\mathrm{P}<0.01$ & $4.56(2.45-8.49)$ & \\
\hline \multicolumn{5}{|c|}{ WHO clinical stage } \\
\hline Stage I & 1 & & 1 & \multirow{4}{*}{$\mathrm{P}<0.01$} \\
\hline Stage II & $1.08(1.04-1.12)$ & $\mathrm{P}<0.01$ & 1.18(1.13-1.23) & \\
\hline Stage III & $1.15(1.12-1.19)$ & $\mathrm{P}<0.01$ & $1.00(0.97-1.04)$ & \\
\hline Stage IV & $1.00(0.95-1.06)$ & $\mathrm{P}<0.01$ & $0.97(0.92-1.02)$ & \\
\hline \multicolumn{5}{|c|}{ ARV groups } \\
\hline No ART & & & 1 & \\
\hline ART & & & $2.23(1.87-2.65)$ & $\mathrm{P}<0.01$ \\
\hline \multicolumn{5}{|c|}{ Enrolment Year } \\
\hline 2012 & 1 & & 1 & \\
\hline 2013 & $1.13(1.09-1.18)$ & $\mathrm{P}<0.01$ & $1.18(1.13-123)$ & \\
\hline 2014 & $1.45(1.40-1.51)$ & $\mathrm{P}<0.01$ & 1.54(1.48-1.60) & \\
\hline
\end{tabular}




\begin{tabular}{|c|c|c|c|c|}
\hline 2015 & $1.87(1.79-1.94)$ & $\mathrm{P}<0.01$ & 2.02(1.94-2.11) & \multirow[t]{2}{*}{$\mathrm{P}<0.01$} \\
\hline 2016 & $2.37(2.27-2.47)$ & $\mathrm{P}<0.01$ & 2.92(2.79-3.06) & \\
\hline \multicolumn{5}{|c|}{ Health Facility type } \\
\hline Dispensary & 1 & & 1 & $\mathrm{P}<0.01$ \\
\hline Health Center & $1.14(1.10-1.18)$ & $\mathrm{P}<0.01$ & $1.19(1.15-1.24)$ & \\
\hline Hospital & $1.74(1.69-1.80)$ & $\mathrm{P}<0.01$ & $1.84(1.77-1.90)$ & \\
\hline \multicolumn{5}{|c|}{ Health Facility ownership } \\
\hline Private & 1 & & 1 & $\mathrm{P}<0.01$ \\
\hline Public & $2.08(2.00-2.15)$ & $\mathrm{P}<0.01$ & 1.82(1.75-1.90) & \\
\hline \multicolumn{5}{|l|}{ Region } \\
\hline Dar es Salaam & 1 & & 1 & \multirow[b]{3}{*}{$\mathrm{P}<0.01$} \\
\hline Iringa & $0.36(0.34-0.38)$ & $\mathrm{P}<0.01$ & $0.44(0.41-0.47)$ & \\
\hline Njombe & $0.80(0.78-0.83)$ & $\mathrm{P}<0.01$ & $0.73(0.71-0.76)$ & \\
\hline \multicolumn{5}{|c|}{ ARV adherence } \\
\hline Poor & 1 & & 1 & \multirow[b]{2}{*}{$\mathrm{P}<0.01$} \\
\hline Good & $0.51(0.43-0.60)$ & $\mathrm{P}<0.01$ & $0.56(0.47-0.67)$ & \\
\hline \multicolumn{5}{|l|}{ Visit number } \\
\hline First visit & 1 & & 1 & \multirow{3}{*}{$\mathrm{P}<0.01$} \\
\hline Within $1^{\text {st }}$ year & $3.86(3.33-4.47)$ & $\mathrm{P}<0.01$ & $2.83(2.19-3.67)$ & \\
\hline $\begin{array}{l}\text { Beyond } 1^{\text {st }} \\
\text { Year }\end{array}$ & $9.00(7.81-10.37)$ & $\mathrm{P}<0.01$ & \begin{tabular}{|c|c|}
$6.77(5.25-8.73)$ \\
\end{tabular} & \\
\hline
\end{tabular}

*95\% Confidence Interval

\section{REFERENCES:}


1. UN Joint Programme on HIV/AIDS (UNAIDS). to help end the AIDS epidemic. United Nations. 2014;

2. WHO | Global tuberculosis report 2018. 2018.

3. Dravid A, Natarajan K, Medisetty M, Gawali R, Mahajan U, Kulkarni M, et al. Incidence of tuberculosis among HIV infected individuals on long term antiretroviral therapy in private healthcare sector in Pune, Western India. BMC Infect Dis. 2019;19(1):1-12.

4. Getahun H, Granich R, Sculier D, Gunneberg C, Blanc L, Nunn P, et al. Implementation of isoniazid preventive therapy for people living with HIV worldwide: Barriers and solutions. Aids. 2010;24(SUPPL. 5).

5. Yirdaw KD, Teklu AM, Mamuye AT, Zewdu S. Breakthrough tuberculosis disease among people with HIV - Should we be worried? A retrospective longitudinal study. PLoS One. 2019;14(2):1-11.

6. Golub J et al. IPT, ARV and TB risk in PLHIV SA study. 2011;23(5):631-6.

7. Shayo GA, Chitama D, Moshiro C, Aboud S, Bakari M, Mugusi F. CostEffectiveness of isoniazid preventive therapy among HIV-infected patients clinicaly screened for latent tuberculosis infection in Dar es Salaam, Tanzania: A prospective Cohort study. BMC Public Health. 2017;18(1):1-9.

8. Organization WH, UNAIDS. Policy statement on preventive therapy against tuberculosis in people living with HIV. Report of a meeting held in Geneva 18 20 February 1998. WHO Doc. 1998;WHO/TB/98.(February):1-26.

9. Lester R, Hamilton R, Charalambous S, Dwadwa T, Chandler C, Churchyard GJ, et al. Barriers to implementation of isoniazid preventive therapy in HIV clinics: A qualitative study. Aids. 2010;24(SUPPL. 5):45-8.

10. Grant AD, Charalambous S, Fielding KL, Day JH, Corbett EL, Chaisson RE, et al. Effect of routine isoniazid preventive therapy on tuberculosis incidence among 
HIV-infected men in South Africa: A novel randomized incremental recruitment study. J Am Med Assoc. 2005;293(22):2719-25.

11. Ayele HT, van Mourik MSM, Bonten MJM. Effect of isoniazid preventive therapy on tuberculosis or death in persons with HIV: A retrospective cohort study. BMC Infect Dis. 2015;15(1):1-8.

12. Makoni A, Chemhuru M, Tshimanga M, Gombe NT, Mungati M, Bangure D. Evaluation of the isoniazid preventive therapy (IPT) program in Shurugwi District, Midlands Province, Zimbabwe, January 2013 to August 2014. BMC Res Notes. 2015;8(1):4-9.

13. Wilkinson D, Davies GR. The increasing burden of tuberculosis in rural South Africa - Impact of the HIV epidemic. South African Med J. 1997;87(4):447-50.

14. Mindachew M, Deribew A, Memiah P, Biadgilign S. Perceived barriers to the implementation of isoniazid preventive therapy for people living with HIV in resource constrained settings: A qualitative study. Pan Afr Med J. 2014;17:1-6.

15. Sabasaba A, Mwambi H, Somi G, Ramadhani A, Mahande MJ. Effect of isoniazid preventive therapy on tuberculosis incidence and associated risk factors among HIV infected adults in Tanzania: A retrospective cohort study 11 Medical and Health Sciences 1117 Public Health and Health Services. BMC Infect Dis. 2019;19(1):1-8.

16. URT. Tanzania Hiv Impact Survey (THIS) 2016-2017. Tanzania HIV Impact Surv 2016-2017. 2018;(December 2017):2016-7.

17. Said K, Hella J, Mhalu G, Chiryankubi M, Masika E, Maroa T, et al. Diagnostic delay and associated factors among patients with pulmonary tuberculosis in Dar es Salaam, Tanzania. Infect Dis Poverty. 2017;6(1):1-10.

18. Maokola W, Ngowi B, Lawson L, Mahande M, Todd J, Msuya S. Performance of and Factors Associated With Tuberculosis Screening and Diagnosis Among 
People Living With HIV: Analysis of 2012-2016 Routine HIV Data in Tanzania. Front Public Heal. 2020;7(404).

19. Thindwa D, Macpherson P, Choko AT, Khundi M, Sambakunsi R, Ngwira LG, et al. Completion of isoniazid preventive therapy among human immunodeficiency virus positive adults in urban Malawi. Int J Tuberc Lung Dis. 2018;22(3):273-9.

20. Lewis JJ, Fielding KL, Grant AD, Chihota VN, Popane F, Luttig M, et al. Eligibility for isoniazid preventive therapy in South African gold mines. PLoS One. $2013 ; 8(11): 1-6$.

21. Nyathi S, Dlodlo RA, Satyanarayana S, Takarinda KC, Tweya H, Hove S, et al. Isoniazid preventive therapy: Uptake, incidence of tuberculosis and survival among people living with HIV in Bulawayo, Zimbabwe. PLoS One. 2019;14(10):1-12.

22. Teklay G, Teklu T, Legesse B, Tedla K, Klinkenberg E. Barriers in the implementation of isoniazid preventive therapy for people living with HIV in Northern Ethiopia: A mixed quantitative and qualitative study. BMC Public Health. 2016;16(1):1-9.

23. Wesen A, Mitike G. Provision and awareness for isoniazid preventive therapy among PLHIV in Addis Ababa, Ethiopia. BMC Int Health Hum Rights. 2012;12(1):2.

24. Dhungana GP, Thekkur P, Chinnakali P, Bhatta U, Pandey B, Zhang WH. Initiation and completion rates of isoniazid preventive therapy among people living with HIV in Far-Western Region of Nepal: A retrospective cohort study. BMJ Open. 2019;9(5):1-9.

25. Wesen Denegetu A, Lovely Dolamo B. Tuberculosis case finding and isoniazid preventive therapy among people living with HIV at public health facilities of Addis Ababa, Ethiopia: a cross-sectional facility based study. BMC Public 
Health. 2014;14(1):1-15.

26. Trinh TT, Han DT, Bloss E, Le TH, Vu TT, Mai AH, et al. HHS Public Access. 2016;109(10):653-9.

27. Van Ginderdeuren E, Bassett J, Hanrahan C, Mutunga L, Van Rie A. Health system barriers to implementation of TB preventive strategies in South African primary care facilities. PLoS One. 2019;14(2):1-12.

28. Ayele AA, Atnafie SA, Balcha DD, Weredekal AT, Woldegiorgis BA, Wotte MM, et al. Self-reported adherence and associated factors to isoniazid preventive therapy for latent tuberculosis among people living with HIV/AIDS at health centers in Gondar town, North West Ethiopia. Patient Prefer Adherence. 2017;11:743-9.

29. Buregyeya E, Rutebemberwa E, LaRussa P, Lal S, Clarke SE, Hansen KS, et al. Comparison of the capacity between public and private health facilities to manage under-five children with febrile illnesses in Uganda. Malar J. 2017;16(1):1-7. 


\section{Figures}

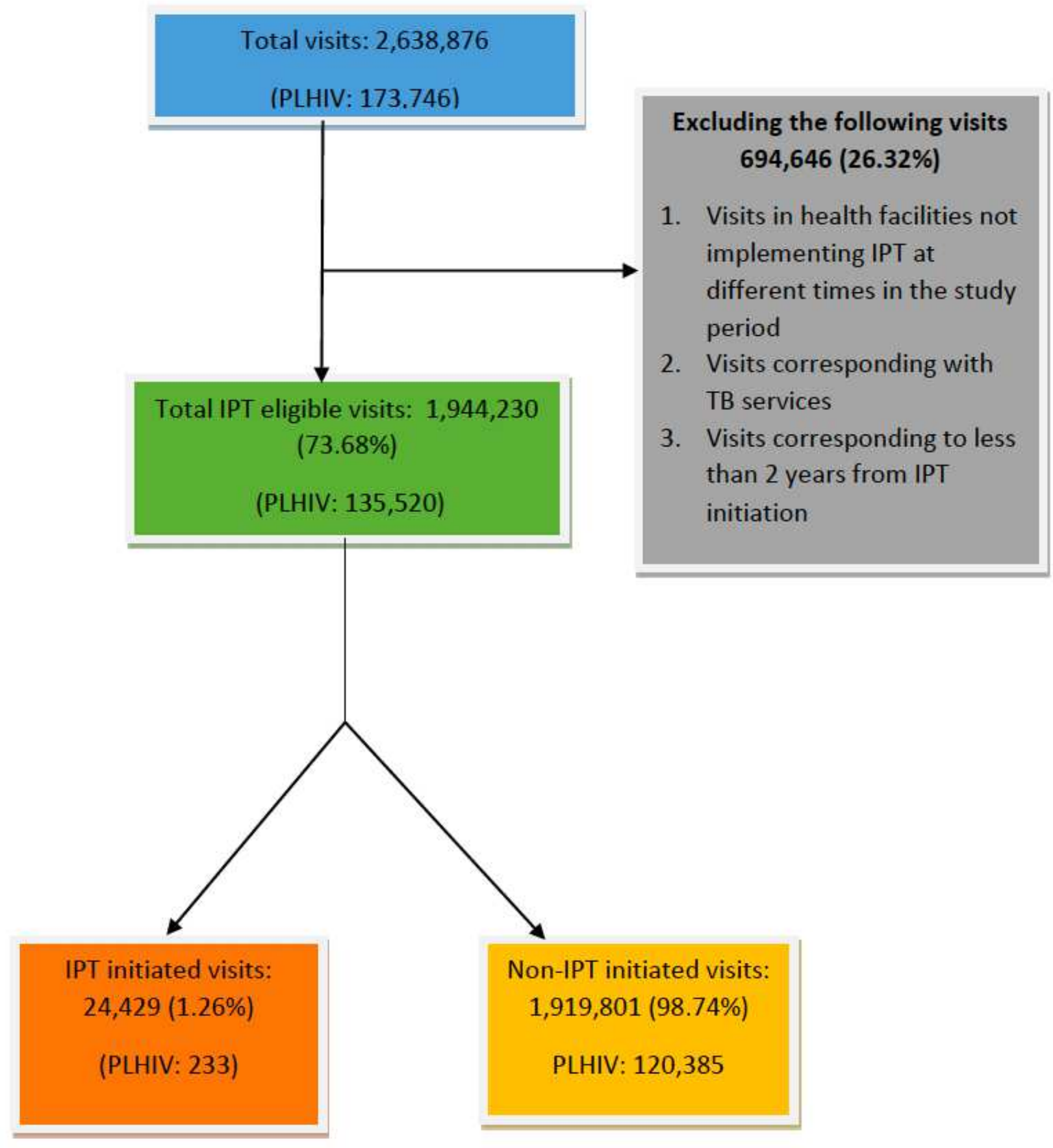

Figure 1

IPT initiation cascade among PLHIV attending CTC during the follow up period 